\title{
Stress no cotidiano da equipe de enfermagem e sua correlação com o cronótipo
}

\author{
Stress resulting from the daily activities of the nursing \\ team and correlation with chronotype
}

\author{
Luciane Ruiz Carmona FERREIRA' \\ Milva Maria Figueiredo DE MARTINO'
}

\begin{abstract}
Resumo
Este estudo teve como propósito classificar o cronótipo dos funcionários da equipe de enfermagem e identificar a presença de stress correlacionado ao cronótipo e turno de trabalho. Foi realizado em hospital privado da cidade de Limeira, estado de São Paulo, com 87 sujeitos, dos diferentes setores e turnos de trabalho. Os instrumentos utilizados foram o Inventário de Sintomas de Stress de Lipp e o Questionário de Indivíduos Matutinos e Vespertinos. Verificou-se que 74,7\% dos sujeitos estavam adequados ao turno de trabalho; no entanto, 55,4\% apresentavam sintomas de stress, demonstrando correlação estatisticamente significativa (Teste Exato de Fisher $p=0,035)$. Verificou-se também stress nos sujeitos com maior tempo de serviço na instituição, com diferença significativa (Teste Exato de Fisher $p=0,003$ ). Concluiu-se que a maioria dos sujeitos estava adequada ao turno de trabalho, de acordo com seu cronótipo, porém mesmo nestes indivíduos o stress foi observado, levando à confirmação de dados obtidos em outras pesquisas quanto ao caráter estressante da profissão.
\end{abstract}

Unitermos: Enfermagem. Stress. Trabalho por turno.

\begin{abstract}
The purpose of present study was to classify the chronotype of nursing staff, in order to identify the presence of stress and its correlation with chronotype and working hours. The study was conducted at a private hospital in the city of Limeira, in the state of São Paulo, with 87 subjects from differentsectors and work shifts. The instruments used were: Lipp's Stress Symptom Inventory and a self-assessment questionnaire to determine morningness-eveningness in human circadian rhythms. We ascertained that $74.7 \%$ of the individuals were acclimated to shift working; however, $55.4 \%$ of them presented symptoms of stress - thereby demonstrating a significant statistical correlation (Fisher's Exact Test, $p=0.035$ ). We also witnessed stress in subjects with a greater length of service in the institution, with a significant difference (Fisher's Exact Test, $p=0.003$ ). Most subjects were acclimated to their work shifts, in accordance with their personal chronotype, nevertheless even with these individuals stress could be observed, leading to the confirmation of data obtained in other research as to the stressful nature of the profession.
\end{abstract}

Uniterms: Nurses. Stress. Shift work.

Na área da saúde, a terminologia stress foi utilizada pela primeira vezem 1936, pelo médico endocrinologista Hans Selye, quando ainda cursava a Faculdade de
Medicina e sentia-se incomodado ao perceber que havia algo em comum entre os seres humanos doentes, independentemente da doença existente, denominando

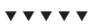

1 Universidade Estadual de Campinas, Faculdade de Ciências Médicas, Departamento de Enfermagem, Curso de Pós-Graduação. R. Tessalia Vieira de Camargo, 126, Cidade Universitária, 13084-971, Campinas, SP, Brasil. Correspondência para/Correspondence to: L.R.C. FERREIRA. E-mail: <luruiz@fcm.unicamp.br>.
} 
esse conjunto de sintomas como Síndrome de Estar Doente (Albrecht, 1990).

A ideia do caráter exclusivamente negativo do stress foi abandonada quando Selye (1959) relatou que ele não se origina apenas como resposta a uma ameaça ou agente agressor, mas também pode resultar de situações agradáveis, saudáveis e necessárias, como uma promoção no emprego, o casamento ou o nascimento de um filho. Para designar o stress decorrente destas situações agradáveis, o pesquisador atribuiu o termo eustress, e para as situações contrárias, o termo distress.

Vários outros autores fazem menção ao caráter positivo e necessário do stress, como Molina (1996), que o definiu como qualquer tensão, aguda ou crônica, capaz de produzir mudança de comportamento físico e emocional, e uma resposta de adaptação psicofisiológica que pode ser negativa ou positiva para o organismo.

As manifestações orgânicas frente ao stress podem ser observadas tanto na forma física como na psicológica, sendo mais comuns, entre as físicas, o aumento da sudorese, "nó no estômago", taquicardia, tensão muscular, hipertensão arterial, hiperatividade, mãos e pés frios, náuseas; e entre as psicológicas, ansiedade, tensão, angústia, insônia, alienação, dificuldade de relacionamento, preocupação excessiva, incapacidade de concentração, tédio, ira, depressão e hipersensibilidade emotiva (Pafaro, 2004).

Após longo período de pesquisa, Selye (1959) definiu o stress como um estado manifestado por uma síndrome específica, embora constituída por alterações inespecíficas, que são produzidas num sistema biológico, dividindo-o em três fases:

1) Fase de alarme ou alerta: referente ao momento inicial, em que o organismo identifica o estressor e mobiliza uma resposta orgânica rápida para o enfrentamento;

2) Fase de resistência: nesta fase ocorre o aumento de resistência do organismo, independentemente da permanência ou não do estressor, com adaptação do organismo: a respiração, os batimentos cardíacos, a circulação e a pressão arterial voltam a níveis anteriores;

3) Fase de exaustão: o estressor permanece e o organismo não é capaz de eliminá-lo ou adaptar-se 66 adequadamente.
Embora Selye tenha dividido a Síndrome de Adaptação Geral em três etapas, em estudo recente Lipp (2000) identificou uma quarta fase, que se desenvolve entre a fase de resistência e exaustão, denominada fase de quase-exaustão. Nesta fase há enfraquecimento e incapacidade do indivíduo em resistir ou adaptar-se ao estressor, podendo surgir leves problemas de saúde, que não o incapacitam.

Altos níveis de stress que se mantêm contínuos, além da possibilidade de desencadearem doenças físicas, podem gerar um quadro de esgotamento emocional, caracterizado por sentimentos negativos como pessimismo, atitudes desfavoráveis em relação ao trabalho, mudança de comportamento com os colegas, não aceitação de novas informações e resolução superficial de problemas (Lipp \& Tanganelli, 2002; Pafaro \& De Martino, 2004). Esse desgaste causado pelo stress pode levar o indivíduo ao estado de Burnout, termo muito usado atualmente nos Estados Unidos, que descreve uma realidade de stress crônico em profissionais que desenvolvem atividades que exigem um alto grau de contato com as pessoas (Lipp \& Tanganelli, 2002).

Muitas vezes o indivíduo reconhece o fenômeno do stress e quais suas causas, porém não se interessa por eliminá-lo, ou então se sente impossibilitado para tal. Entre os inúmeros profissionais que fazem dupla jornada de trabalho e ainda cumprem com seus afazeres domésticos, cuidam da educação dos filhos e auxiliam no orçamento para a manutenção da família, encontram-se os da área de enfermagem (Pafaro \& De Martino, 2004).

Nota-se que as jornadas de trabalho estão cada vez mais longas, gerando uma série de novos problemas relacionados ao trabalho em turnos. Estudos realizados mostraram que o trabalho noturno é a causa mais comum de alterações na saúde do trabalhador (De Martino \& Cipolla-Neto, 1999).

\section{Cronobiologia}

A cronobiologia é a ciência que estuda a organização temporal dos fenômenos biológicos, fisiológicos e/ou psicológicos, que permite a compreensão de que o organismo é fisiologicamente diferente a cada momento do dia, com capacidade diferente de reagir aos estímulos ambientais, sejam eles físicos, químicos, biológicos ou sociais (Marques \& Menna-Barreto, 1999). 
Segundo Horne e Ostberg (1976), os indivíduos podem ser classificados em três cronótipos, considerando as diferenças individuais de adaptação temporal dos ritmos biológicos. Os cronótipos são:

- Matutino (dividido em tipos extremo e moderado): indivíduos que preferem dormir cedo (em torno das 21 ou 22 horas) e também acordam cedo, em torno das seis horas, sem dificuldades, estando já nesse momento perfeitamente aptos para o trabalho, com bom nível de alerta e bom desempenho físico e mental pela manhã;

- Vespertino (dividido em tipos extremo e moderado): preferem dormir e acordar tarde (em torno de uma hora da manhã e após as 10 horas, respectivamente), com melhor disposição e desempenho no período da tarde e início da noite;

- Indiferente indivíduos que têm maior flexibilidade, escolhendo horários intermediários de acordo com as necessidades de sua rotina.

Assim, mostra-se de grande relevância o conhecimento do cronótipo (características de matutinidade/ vespertinidade) dos trabalhadores, a fim de auxiliar a definir períodos de melhor desempenho físico e mental, o que influencia as atividades de um modo geral (De Martino \& Ling, 2004).

O que ocorre nos serviços de saúde, muitas vezes, é a falta de interesse nos aspectos relativos à promoção e à manutenção da saúde física, mental e social do profissional que atua nessas instituições. Cabe então a esta ciência, a cronobiologia, elucidar questões relativas à tolerância ao trabalho, ou seja, ao processo de ajuste dos indivíduos a uma nova situação temporal imposta pelo esquema de trabalho.

Desta forma, este estudo teve como objetivos: Identificar a presença do stress e suas correlações com o cronótipo e horário de trabalho; classificar o cronótipo dos funcionários da equipe de enfermagem, de acordo com o turno de trabalho; propor medidas adequadas para prevenir o stress e melhorar a qualidade de cuidados assistenciais de enfermagem.

\section{Método}

A pesquisa foi desenvolvida em um hospital privado na cidade de Limeira (SP). O hospital possui 75 leitos. A amostra foi composta por 87 profissionais: enfermeiros, técnicos e auxiliares de enfermagem, dos diferentes turnos de trabalho, dos setores de Pronto-Atendimento, Centro Cirúrgico, Clínica Médica e Cirúrgica, Maternidade, Pediatria, Unidade Neonatal e Unidade de Terapia Intensiva de Adulto.

O regime de trabalho existente consta de jornadas de seis horas diárias para os períodos matutino e vespertino, com uma folga semanal, e para o noturno é de 12 horas de trabalho, com descanso de 36 horas e duas folgas mensais. A faixa etária dos participantes esteve compreendida entre 19 e 51 anos; a população feminina foi predominante.

Os instrumentos utilizados para a coleta dos dados foram os seguintes: ficha de Identificação; inventário de Sintomas de Stress de Lipp (ISS-LIPP) (Lipp, 2000) e questionário de Identificação de Indivíduos Matutinos e Vespertinos (Horne \& Ostberg, 1976).

\section{Procedimentos}

Inicialmente foi distribuído a todos os funcionários da equipe de enfermagem um documento explicando sobre a pesquisa na instituição e a importância da participação de cada um. Após confirmação da participação de cada sujeito, foi entregue um Termo de Consentimento Livre e Esclarecido, de acordo com a aprovação do Comitê de Ética, sob parecer n³74/2004.

Em seguida iniciou-se a coleta dos dados, primeiramente respondendo ao questionário para identificação do cronótipo e o Inventário de Sintomas do Stress.

O horário da coleta dos dados foi durante o período de trabalho dos funcionários, ou seja, nos turnos da manhã, tarde e noite. O próprio pesquisador entregou os instrumentos, fornecendo orientações sobre o preenchimento e esclarecendo as dúvidas encontradas.

A análise estatística dos resultados obtidos na pesquisa foi estabelecida por meio da utilização de tabelas de frequência das variáveis categóricas e estatísticas descritivas das variáveis contínuas; para tal utilizaram-se os testes: Qui-Quadrado, Exato de Fisher e Mann-Withney. O nível de significância adotado para os testes estatísticos foi de 5\%, ou seja, $p<0,05$. 
Os aspectos éticos foram respeitados, conforme a Resolução 196/96 do Conselho Nacional de Saúde, referente às recomendações para pesquisas com seres humanos. Além disso, antes do início da coleta de dados o projeto foi submetido a análise pela Comissão de Ética em Pesquisa da Faculdade de Ciências Médicas, onde foi aprovado.

A correção e interpretação dos Inventários de Sintomas de Stress de Lipp foram realizadas por uma psicóloga, conforme solicita o Conselho Federal de Psicologia.

\section{Resultados e Discussão}

Dos 87 sujeitos que participaram da pesquisa, 71 (81,6\%) eram do sexo feminino e 16 eram $(18,4 \%)$ do sexo masculino. Este fato pode estar relacionado à origem da profissão de enfermagem, que sempre esteve ligada à noção de cuidar, ou seja, mulheres prestando cuidados aos que necessitam e favorecendo a sobrevivência das pessoas (Tabela 1).

Quanto à idade, na amostra estudada, 87,4\% dos profissionais tinham entre 20 e 39 anos de idade, ou seja, eram adultos jovens, em idade de significativa capacidade produtiva, sendo esta vitalidade confirmada pelo fato de a maioria dos sujeitos possuir outra atividade além do trabalho na referida instituição.

Entre os casados e com união estável, 92,9\% dos companheiros trabalhavam, auxiliando na manutenção do lar. Tal fato talvez possa ser justificado devido aos baixos salários dos profissionais da enfermagem, o que impõe a necessidade de que os parceiros também trabalhem.

Constatou-se que $79,3 \%$ dos profissionais possuíam nível médio completo e que a maioria atuava nos cargos de auxiliar $(39,1 \%)$ e técnico de enfermagem (48,3\%), ou seja, mantinham contato direto e prolongado com os pacientes, promovendo desgaste físico e psicológico ao profissional.

Quanto ao tempo de trabalho na instituição pesquisada, por ter sido recentemente inaugurada, $66,7 \%$ dos sujeitos tinham período de um a dois anos de trabalho. Ao contrário do que tem sido encontrado em outras pesquisas, nas quais a maioria da população faz
Tabela 1. Características gerais da amostra estudada $(n=87)$. Limeira (SP), 2005

\begin{tabular}{|c|c|c|}
\hline & Frequência & Porcentagem \\
\hline \multicolumn{3}{|l|}{ Sexo } \\
\hline Masculino & 16 & 18,4 \\
\hline Feminino & 71 & 81,6 \\
\hline \multicolumn{3}{|l|}{ Idade } \\
\hline$<20$ anos & 3 & 3,4 \\
\hline 20-29 anos & 56 & 64,4 \\
\hline 30-39 anos & 20 & 23,0 \\
\hline 40-49 anos & 7 & 8,0 \\
\hline$>50$ anos & 1 & 1,1 \\
\hline \multicolumn{3}{|l|}{ Estado civil } \\
\hline Solteiro & 40 & 46,0 \\
\hline Casado & 40 & 46,0 \\
\hline União estável & 2 & 2,3 \\
\hline Separado & 5 & 5,7 \\
\hline \multicolumn{3}{|l|}{ Escolaridade } \\
\hline $1^{\circ} \mathrm{grau}$ & 3 & 3,4 \\
\hline $2^{\circ} \mathrm{grau}$ & 69 & 79,3 \\
\hline Superior & 15 & 17,2 \\
\hline \multicolumn{3}{|l|}{ Cargo } \\
\hline Auxiliar & 34 & 39,1 \\
\hline Técnico & 42 & 48,3 \\
\hline Enfermeiro & 11 & 12,6 \\
\hline \multicolumn{3}{|c|}{ Tempo na instituição } \\
\hline$<1$ ano & 22 & 25,3 \\
\hline $1-2$ anos & 58 & 66,7 \\
\hline$>2$ anos & 7 & 8,0 \\
\hline \multicolumn{3}{|c|}{ Turno de trabalho } \\
\hline Manhã & 29 & 33,3 \\
\hline Tarde & 19 & 21,8 \\
\hline Noite & 29 & 33,3 \\
\hline Manhã/Tarde & 9 & 10,3 \\
\hline Tarde/Noite & 1 & 1,1 \\
\hline \multicolumn{3}{|l|}{ Dupla jornada } \\
\hline Não & 49 & 56,3 \\
\hline Sim & 38 & 43,7 \\
\hline
\end{tabular}

dupla jornada e atribui isto à necessidade econômica devida aos baixos salários da categoria de enfermagem (Pafaro \& De Martino, 2004; Vieira, 2001), neste estudo $56,3 \%$ referiram não possuir outro trabalho.

A Tabela 2 mostra a adequação dos trabalhadores nos turnos de trabalho e respectivos cronótipos. Verificou-se que os indivíduos moderadamente matutinos trabalhavam, em sua maioria, no período da manhã (45,7\%), e os indiferentes encontravam-se principalmente no turno da noite $(35,7 \%)$. 
Tabela 2. Identificação de adequação do cronótipo versus turno de trabalho. Limeira (SP), 2005.

\begin{tabular}{lcc}
\hline Identificação & Frequência & Porcentagem \\
\hline Adequado & 65 & 74,7 \\
Inadequado & 22 & 25,3 \\
\hline Total & 87 & 100,0 \\
\hline
\end{tabular}

Tabela 3. Avaliação da incidência de stress nos sujeitos. Limeira (SP), 2005.

\begin{tabular}{lcc}
\hline & Frequência & $\%$ \\
\hline Sim & 48 & 55,2 \\
Não & 39 & 44,8 \\
\hline Total & 87 & 100,0 \\
\hline
\end{tabular}

Devido a esses achados, constatou-se que existia adequação entre o turno de trabalho e seu respectivo cronótipo: 74,7\% dos indivíduos estavam devidamente alocados. No entanto, não havia avaliação cronobiológica prévia à contratação; os funcionários eram fixados no plantão conforme a vaga disponível.

Entre os que relataram praticar dupla jornada de trabalho, verificou-se que apenas $44,7 \%$ dos sujeitos apresentavam sintomas de stress, possivelmente por estarem já adaptados à rotina, ou por se sentirem seguros financeiramente praticando a dupla jornada.

Quanto à classificação dos cronótipos, observou-se que as porcentagens foram de 48,3\% para os indivíduos do tipo indiferente e $40,2 \%$ para os moderadamente matutinos.

Os indivíduos do tipo indiferente, conforme explicado, são aqueles que têm maior flexibilidade, ou seja, acordar mais ou menos cedo é indiferente, demonstrando capacidade de se ajustar conforme as necessidades de sua rotina. Estes constituem a maior parte da população, e não apenas a de profissionais da enfermagem (Campos \& De Martino, 2001; De Martino \& Ceolim, 2001).

Embora tenha sido observado o predomínio de indivíduos do tipo indiferente, com a maioria da amostra adequada ao turno de trabalho, verificou-se que 55,2\% dos indivíduos apresentavam sintomas de stress, conforme mostra a Tabela 3. Esta incidência pode ser justificada por ser a enfermagem uma profissão estressante, classificada pela Health Education Authority como a quarta profissão mais estressante do setor público (Murofuse Abranches \& Napoleão, 2005).

Essa característica da enfermagem tem sido relacionada à história da profissão, que revela problemas que surgiram já no início de sua implementação no Brasil e em outros países e que ainda hoje são latentes, como a marginalização, que leva o enfermeiro a buscar constantemente sua afirmação profissional frente a outros profissionais. Além disso, existem vários outros problemas que não podem ser esquecidos na dimensão do stress relacionado à enfermagem, como o número reduzido de enfermeiros na equipe, a falta de reconhecimento profissional e os baixos salários, que levam o indivíduo a atuar em mais de um local de trabalho, desempenhando uma extensa carga horária mensal (Carmelo \& Angerami, 2004; Farias, Mauro \& Zeitoune, 2000; Murofuse et al., 2005; Stacciarini \& Tróccoli, 2001).

Entre os sujeitos com stress, foi verificado que $83,3 \%$ encontravam-se na fase de resistência, com prevalência dos sintomas psicológicos. Destes, 27,1\% estavam em estado de agravamento.

Diferentes pessoas podem reagir a um mesmo estressor de maneira diferente, ou seja, a capacidade de lidar com os eventos estressores pode variar conforme a herança genética, estilo de vida, estratégias de enfrentamento utilizadas pelo indivíduo, bem como a experiência adquirida durante a vida (Lipp, 1996; Murta \& Tróccoli, 2004).

O stress também foi verificado em elevadas porcentagens entre as mulheres, com 52,1\%. Esta elevada incidência entre as mulheres pode estar relacionada ao fato de, além de trabalharem na instituição hospitalar, terem que desenvolver outra jornada em casa, cumprindo com os afazeres domésticos e cuidando da educação dos filhos. Somados a isso, deve-se lembrar das condições de trabalho a que estas profissionais estão submetidas, as quais muitas vezes não condizem com as exigências para o desenvolvimento de suas atividades. Outra questão é o fato de a equipe de enfermagem conviver diariamente com situações indutoras de stress, principalmente por lidar com a dor, sofrimento e morte, podendo gerar exaustão emocional (Chang, Hancock, Johnson, Daly \& Jackson, 2005; Jenkins \& Elliot, 2004; Stacciarini \&Tróccoli, 2001). 
Por ser o stress um tema tão difundido atualmente, e com dados alarmantes, vários têm sido os estudos realizados, inclusive com profissionais da área da saúde, recebendo destaque aqueles que compõem a equipe de enfermagem (Carmelo \& Angerami, 2004; Chang et al., 2005; Ferreira, 1998; Jenkins \& Elliot, 2004) e os enfermeiros (Miranda, 1998; Pafaro \& De Martino, 2004; Stacciarini \&Tróccoli, 2001; Vieira, 2001). Essas pesquisas buscam entender os fenômenos provocados nos profissionais frente ao stress.

Os achados do estudo aqui descrito confirmaram a tendência estressante do trabalho de enfermagem, presente em 55,6\% dos sujeitos que possuíam ensino fundamental e médio completo e 53,3\% dos que possuíam nível superior.

Para a variável tempo de trabalho na instituição, houve diferença significativa dos resultados: $67,2 \%$ dos sujeitos pesquisados que trabalham no hospital há mais de um ou dois anos apresentaram sintomas de stress. Foi verificado também que $65,5 \%$ dos indivíduos do turno da manhã e 55,2\% do noturno possuíam manifestações de stress. A elevada incidência no turno da manhã pode estar relacionada à rotina intensa do hospital, pois quase todos os procedimentos de enfermagem banhos, curativos, medicações, coleta de exames e

Tabela 4. Avaliação dos sujeitos quanto à presença de stress relacionado ao tempo de trabalho na instituição. Limeira (SP), 2005.

\begin{tabular}{lccccc}
\hline & \multicolumn{3}{c}{ Não } & & \multicolumn{2}{c}{ Sim } \\
\cline { 2 - 3 } \cline { 6 - 7 } \cline { 6 - 6 } & Frequência & $\%$ & & Frequência & $\%$ \\
\hline$<1$ ano & 16 & 72,73 & & 6 & 27,27 \\
$1-2$ anos & 19 & 32,76 & & 39 & 67,24 \\
$>2$ anos & 4 & 57,14 & & 3 & 42,86 \\
\hline Total & 39 & & & 48 & \\
\hline
\end{tabular}

Teste Exato de Fisher: $p=0,003$. encaminhamento de pacientes ao centro cirúrgico são realizados neste período, além de todos os pacientes receberem visita médica até as 12 horas (Tabela 4).

Quanto à distribuição dos sujeitos com stress nos setores de trabalho, observou-se maior porcentagem entre os enfermeiros que desempenham função de Supervisão da Equipe $(85,7 \%)$, seguidos pelos funcionários do Centro Cirúrgico (71,4\%) e da Enfermaria Térrea (69,2\%) (Tabela 5).

Outro dado importante foi que $52,9 \%$ dos sujeitos que trabalharam de um a dois anos no período noturno, bem como $73,3 \%$ dos que trabalharam mais de dois anos, possuíam sintomas de stress.

É reconhecido que o trabalho noturno altera o ritmo circadiano, provocando stress, que gera, entre outras coisas, alterações no padrão do sono, promovendo uma retroalimentação da situação.

Lida (2001) cita a influência dos turnos na quantidade total de erros observados. Em seu estudo, o noturno destacou-se como o de maior ocorrência de erros. A autora constatou ainda que o desempenho de um trabalhador do turno da noite é comparável ao de um trabalhador diurno que tenha passado a noite inteira sem dormir. O sono diurno não possui a mesma qualidade e capacidade reparadora do sono noturno, gerando nos trabalhadores deste período um débito de sono cumulativo, que resulta em diminuição da capacidade produtiva.

Quando avaliada a distribuição dos sujeitos conforme o cronótipo no turno de trabalho, verificou-se que os do tipo matutino são os que se encontram melhor alocados, no turno da manhã. Viu-se também que $100 \%$ do tipo vespertino e $35 \%$ dos classificados como indiferentes estão no turno da noite. Nestas variáveis houve correlação significativa pelo teste Exato de Fisher, $\operatorname{com} p=0,035$.

Tabela 5. Distribuição dos sujeitos de acordo com o cronótipo e turno de trabalho. Limeira (SP), 2005.

\begin{tabular}{|c|c|c|c|c|c|c|c|c|c|}
\hline \multirow{2}{*}{ Cronótipo } & \multicolumn{2}{|c|}{ Manhã } & \multicolumn{2}{|c|}{ Tarde } & \multicolumn{2}{|c|}{ Noite } & \multicolumn{2}{|c|}{ Misto } & \multirow{2}{*}{ Total } \\
\hline & Frequência & $\%$ & Frequência & $\%$ & Frequência & $\%$ & Frequência & $\%$ & \\
\hline Vespertino & 0 & 0,00 & 0 & 0,00 & 5 & 100,00 & 0 & 0,00 & 5 \\
\hline Indiferente & 11 & 26,19 & 9 & 21,43 & 15 & 35,71 & 7 & 16,67 & 42 \\
\hline Matutino & 18 & 45,00 & 10 & 25,00 & 9 & 22,5 & 3 & 7,50 & 40 \\
\hline Total & 29 & & & & 48 & & & & 87 \\
\hline
\end{tabular}


Para as variáveis turno de trabalho e fase do stress, $100 \%$ dos sujeitos que realizavam turno misto de trabalho (M/T eT/N) encontravam-se em fase de resistência, bem como 93,7\% dos sujeitos do turno noturno, 87,5\% do vespertino e $68,4 \%$ do matutino.

Apenas entre os indivíduos do turno da manhã foram verificados sintomas da fase de alerta, entre os quais $21,1 \%$ dos sujeitos estavam na fase de quaseexaustão.

Entre os indivíduos que estavam adequadamente alocados no turno de trabalho, 80,6\% apresentavam sintomas da fase de resistência e, entre os não adequados, o valor foi de $91,7 \%$ na mesma fase.

Esses achados assemelham-se aos da pesquisa de Pafaro \& De Martino (2004), que avaliou o stress em enfermeiros que atuavam em hospital de oncologia pediátrica. Em seu estudo, verificou-se a prevalência da fase de resistência.

Lipp e Malagris (2001) enfatizam que, na fase de resistência, o organismo busca o reequilíbrio, com uso de grande quantidade de energia para isso, podendo resultar em sensação de desgaste generalizado, aparentemente sem causa. Ressaltam ainda que quanto maior o esforço praticado para adaptação e restabelecimento da harmonia interior, maior é o desgaste sofrido. No entanto, quando o organismo consegue se adaptar completamente, o processo de stress é interrompido e os sintomas desaparecem, não deixando sequelas.

\section{Considerações Finais}

De acordo com os objetivos propostos para esta pesquisa, verificou-se que:

- Houve diferença estatisticamente significativa quanto ao tempo de trabalho na instituição, e no período de um a dois anos houve maior incidência de indivíduos com stress;

- Houve diferença estatisticamente significativa quanto à avaliação da distribuição dos sujeitos nos turnos de trabalho, de acordo com seu cronótipo; $45 \%$ dos sujeitos matutinos estavam alocados no turno da manhã e 35,71\% dos indiferentes estavam no noturno.

Sugere-se que o conhecimento da existência do ritmo biológico pode auxiliar os enfermeiros quanto à organização do serviço de enfermagem: na elaboração de escalas de trabalho, na adequação dos horários às características individuais, na consideração das preferências do sentido de rotação para os turnos de trabalho, na redução dos níveis de stress e na otimização da assistência prestada.

É de grande importância que haja adequação das características do ritmo biológico do trabalhador ao seu horário de trabalho, pois esta medida certamente influenciará decisivamente na qualidade da assistência prestada ao cliente.

\section{Referências}

Albrecht, K. (1990). O gerente e o stress. Rio de Janeiro: JZE Editora.

Campos, M. L. P., \& De Martino, M. M. F. (2001). Estudos das características cronobiológicas dos enfermeiros docentes: cronótipo. Revista Nursing, 11 (42): 31-34.

Carmelo, S. H. H., \& Angerami, E. L. S. (2004). Sintomas de stress nos trabalhadores atuantes em cinco núcleos de saúde da família. Revista Latino-Americana de Enfermagem, 12 (1), 14-21.

Chang, E. M., Hancock, K. M., Johnson, A., Daly, J., \& Jackson, D. (2005). Role stress in nurses: review of related factors and strategies for moving forward. Nursing Health Science, 7 (1), 57-65.

De Martino, M. M. F., \& Cipolla-Neto, J. (1999). Repercussões do ciclo vigília-sono e o trabalho em turnos de enfermeiras. Revista de Ciências Médicas, 3 (8): 81-4.

De Martino, M. M. F., \& Ceolim, M. F. (2001). Avaliação do cronótipo de um grupo de enfermeiros de hospitais de ensino. Revista de Ciências Médicas, 10 (1):19-27.

De Martino, M. M. F., \& Ling, S. Y. (2004). Características cronobiológicas de um grupo de alunos universitários de enfermagem. Revista de Ciências Médicas, 13 (1): 43-49.

Farias, S. N. P., Mauro, M. Y. C., \& Zeitoune, R. C. G. (2000). Questões legais sobre a saúde do trabalhador de enfermagem. Revista Enfermagem UERJ, 8 (1), 28-32.

Ferreira, F. G. (1998). Desvendando o stress da equipe de enfermagem em terapia intensiva. Dissertação de mestrado não-publicada, Escola de Enfermagem, Universidade de São Paulo.

Horne, J. A., \& Ostberg, O. (1976). A self-assesment questionnaire to determine morningness-eveningness in human circadian rhythms. International Journal Chrobiology, 4 (2), 97-110.

Jenkins, R., \& Elliot, P. (2004). Stressors, burnout and social support: nurses acute mental health settings. Journal Advance Nursing, 48 (6), 622-31.

Lida, I. (2001). Ergonomia: projeto e produção. São Paulo: Edgard Blucher. 
Lipp, M. E. N., \& Tanganelli, M. S. (2002). Stress e qualidade de vida em magistrados da justiça do trabalho: diferenças entre homens e mulheres. Psicologia: Reflexão e Crítica, 15 (3), 537-48.

Lipp, M. E. N., \& Malagris, L. E. N. (2001). Manejo do stress. In B. Rangé (Org.), Psicoterapias cognitivo-comportamentais: um diálogo com a psiquiatria. Porto Alegre: Artmed.

Lipp, M. E. N. (1996). Pesquisas sobre stress no Brasil: saúde, ocupações e grupos de risco. Campinas: Papirus.

Lipp, M. E. N. (2000). Manual do Inventário de Sintomas de Stress para Adultos de Lipp (ISSL). São Paulo: Casa do Psicólogo.

Marques, N., \& Menna-Barreto L. (1999). Cronobiologia: princípios e aplicações. São Paulo: Edusp.

Miranda, A. F. (1998). Stress ocupacional: inimigo invisível do enfermeiro? Dissertação de mestrado não-publicada, Escola de Enfermagem de Ribeirão Preto, Universidade de São Paulo, Ribeirão Preto.

Molina, O. F. (1996). Stress no cotidiano. São Paulo: Pancast Editora.

Murofuse, N. T., Abranches, S. S., \& Napoleão, A. A. (2005). Reflexões sobre stress e burnout e a relação com a enfermagem. Revista Latino-Americana de Enfermagem, $13(2), 255-61$.

Murta, S. G., \& Tróccoli, B. T. (2004). Avaliação de intervenção em stress ocupacional. Revista Psicologia:Teoria e Pesquisa, 20 (1), 39-47.

Pafaro, R. C., \& De Martino M. M. F. (2004). Estudo do stress do enfermeiro com dupla jornada de trabalho em um hospital de oncologia pediátrica de Campinas. Revista da Escola Enfermagem USP, 38 (2), 152-60.

Selye, H. (1959). Stress: a tensão da vida. São Paulo: Ibrasa.

Stacciarini, J. M. R., \& Tróccoli, B. T. (2001). O stress na atividade ocupacional do enfermeiro. Revista Latino-Americana Enfermagem, 9 (2), 17-25.

Vieira, L. C. (2001). Stress ocupacional em enfermeiros de um hospital universitário da cidade de Campinas. Dissertação de mestrado não-publicada, Faculdade de Ciências Médicas, Universidade Estadual de Campinas.

Recebido em: 12/2/2008

Versão final reapresentada em: 22/8/2008

Aprovado em: 29/9/2008 